Editorial

\title{
Introduction to a New Open Access Journal by MDPI: Physics
}

\author{
Edward K. Sarkisyan-Grinbaum ${ }^{1,2}$ \\ 1 Experimental Physics Department, CERN, 1211 Geneva 23, Switzerland; \\ Edward.Sarkisyan-Grinbaum@cern.ch \\ 2 Department of Physics, The University of Texas at Arlington, Arlington, TX 76019, USA
}

Received: 31 October 2018; Accepted: 31 October 2018; Published: 2 November 2018

check for updates

Herewith, we launch a new MDPI journal, Physics, for which I am honored to serve as Editor-in-Chief. The journal aims to address all aspects of physics, ranging from fundamental studies to emergent technologies, and promises to be a very interesting publication forum.

The journal aims to consider physics in its complexity, clarifying a full picture of physics, and in this sense connecting different branches and showcasing a diversity of topics under one umbrella. Given the huge significance of physics and its leading contribution to technologies and development in different areas of human activity from everyday use to cosmic investigations, physics plays a crucial role in our progress and in the progress of other sciences. Due to physics' irrevocable contribution to our knowledge, from Aristotle to Isaac Newton and then to Albert Einstein up to nowadays, physics is always at the center of human interest and findings in physics deservedly attract heightened attention.

Considering different aspects of physics, Physics is devoted to jointly publishing searches in fundamental studies and investigations in applied physics, studies on the different areas of physics and on its contribution to modern technologies, and searches spanning from the microworld to the cosmos.

I thank the Members of the Editorial Board for agreeing to serve on this new journal and invite them to contribute their research, as well as inviting papers in order to build a high-quality scientific journal, to seek new horizons and make new discoveries in all areas of physics.

(C) 2018 by the author. Licensee MDPI, Basel, Switzerland. This article is an open access article distributed under the terms and conditions of the Creative Commons Attribution (CC BY) license (http://creativecommons.org/licenses/by/4.0/). 